\title{
Image Registration Method Based On Edge Phase Correlation Algorithm
}

\author{
Qing Zhou ${ }^{1}$, Cheng Yang ${ }^{2}$, Lingyun Wan ${ }^{1}$ and Ying Zhang ${ }^{1}$, Xiaolong

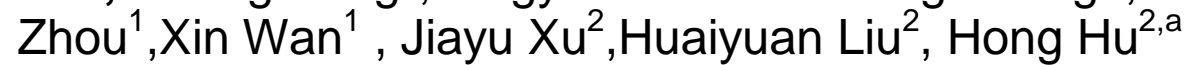 \\ ${ }^{1}$ State Grid Chongqing Electric Power CO. Electric Power Research Institute,Chongqing,China \\ ${ }^{2}$ Harbin Institute of Technology Shenzhen Graduate School, Shenzhen, China \\ a honghu@hitsz.edu.cn
}

Keywords: multiple targets identification; automatic die bonder; reference image

\begin{abstract}
In this paper, a new algorithm being put forward can handle multiple targets identification and location respectively in reference image, with an ability of resistance to rotation, being able to perform angle estimation, and subpixel accuracy location. This paper uses optimized edge phase correlation matching algorithm, with characteristic of remarkable speed, and high accuracy. Experiment shows that this algorithm can be used in automatic die bonder.
\end{abstract}

\section{Introduction}

The purpose of this article is to propose an algorithm which can be used in automatic die bonder. Demand of the algorithm is that it can calculate coordinate and rotation angle of every target in reference image. In ordinary condition, target will not rotate more than 30 degree relative to template. As template image is cut from reference image with all targets having the same size, there is no need to consider scale change. Image from automatic die bonder (as reference image) is usually like Fig.1.

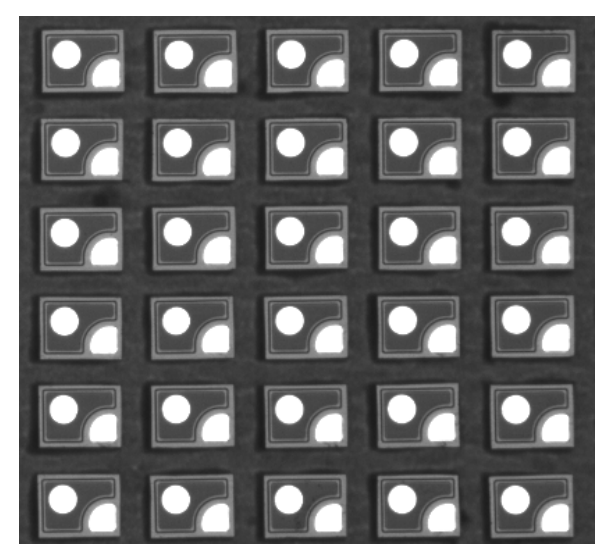

Fig.1. Image taken by automatic die bonder

The algorithm of this article is based on frequency domain. Typical methods are the frequency matching methods, such as cross-correlation based on Fourier transform and phase correlation matching method. Lucchese studied algorithm with characteristics of the rotation resistance, such as the matching problem of translational transformation ${ }^{[2]}$; Reddy put forward a matching algorithm of calculating the rotating transform with FFT method ${ }^{[5]}$; Stone, utilized nonlinear filter and threshold enhancement technique to improve the effect of frequency domain matching algorithm ${ }^{[3]}$; Keller, using pseudo polar Fourier transform (PPFT) in the form of polar coordinates operational, optimizing of the high frequency part of DFT spectrum calculation, improve the performance of image matching greatly ${ }^{[4]}$.

The matching problem between the two images can be summed up as the correlation question ${ }^{[8]}$. So the core issue of image matching can be divided into two aspects: the selection of matching feature and correlation measure. Phase correlation algorithm choosing matching features in frequency domain, with the correlation measure of cross-correlation function, has the characteristics 
of less computation and strong resistance of noise. This method sometimes relies on the translation property of the Fourier transform, which is referred to as the Fourier shift theorem ${ }^{[1]}$. Most of the algorithms using phase correlation algorithm only solve pure translation image registration, without rotation and scale. A portion of the algorithm can solve the problem of spin image.

In this paper, a new registration method, based on phase correlation algorithm, is put forward. First of all, applying optimized edge phase correlation algorithm, this algorithm obtains target areas in reference image roughly, thus changing problem of multiple targets registration into single target registration. Secondly, using Fourier transform, this algorithm can calculate rotate angle between of a chosen target and template. Finally this algorithm compensate rotate angle, i.e., rotate template with calculated angle, reuse edge phase correlation algorithm based on Fourier algorithm, complete the match with translated, rotated targets in reference image.

\section{THEORY}

\section{Phase Correlation Algorithm}

Phase correlation algorithm can only solve image registration problem of pure translation. Set notation of reference image as $\mathrm{R}(\mathrm{x}, \mathrm{y})$, notation of template image as $\mathrm{T}(\mathrm{x}, \mathrm{y})$, they meet the relationship as follow:

$$
\mathrm{R}(\mathrm{x}, \mathrm{y})=\mathrm{T}\left(\mathrm{x}+\mathrm{x}_{0}, \mathrm{y}+\mathrm{y}_{0}\right)
$$

Coordinate $\left(\mathrm{x}_{0}, \mathrm{y}_{0}\right)$ is offset relative to the template to reference image, set $\mathrm{R}_{\mathrm{f}}(\mathrm{u}, \mathrm{v})$ as Fourier transform of reference image $\mathrm{R}(\mathrm{x}, \mathrm{y})$, set $\mathrm{T}_{\mathrm{f}}(\mathrm{u}, \mathrm{v})$ as Fourier transform for the template image $\mathrm{T}(\mathrm{x}, \mathrm{y})$, there are

$$
R_{f}(u, v)=e^{-j 2 \pi\left(u x_{0}+v y_{0}\right)} T_{f}(u, v)
$$

Cross-power spectrum of two images is like:

$$
C C(u, v)=R_{f}(u, v) * T_{f}^{*}(u, v) /\left|R_{f}(u, v) * T_{f}^{*}(u, v)\right|=e^{-j 2 \pi\left(u_{0}+v y_{0}\right)}
$$

Here $T_{f}^{*}(u, v)$ is complex conjugate for $T_{f}(u, v)$, and then perform inverse discrete Fourier transform to $\mathrm{CC}(\mathrm{u}, \mathrm{v})$ to get relation function $\mathrm{CC}(\mathrm{x}, \mathrm{y})$, ideally correlation function is like the pulse function, its peak coordinates is located on offset $\left(\mathrm{x}_{0}, \mathrm{y}_{0}\right)$ between template and reference imaged .

\section{Edge Phase Correlation Algorithm}

However, a performance of FFT with all pixels in both images is time-consuming ${ }^{[9]}$. So thought of less data to handle comes into mind obviously. Apply edge detect towards both images, and utilize edge images to perform FFT can greatly increase the speed of FFT and reduce data to process in complex matrix calculation.

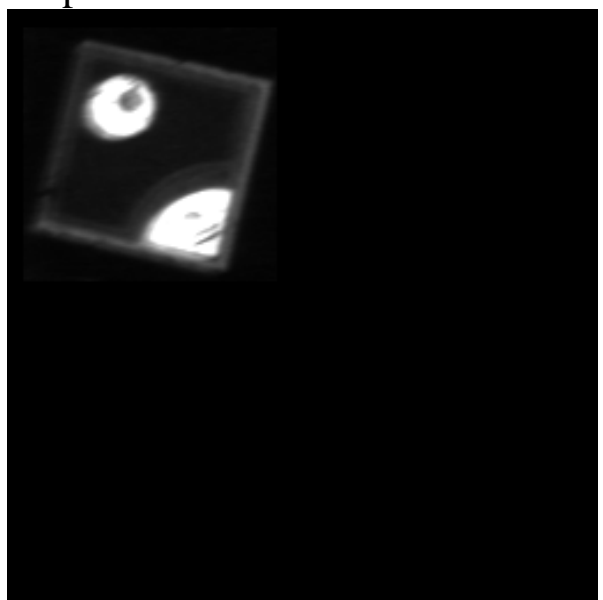

(a)

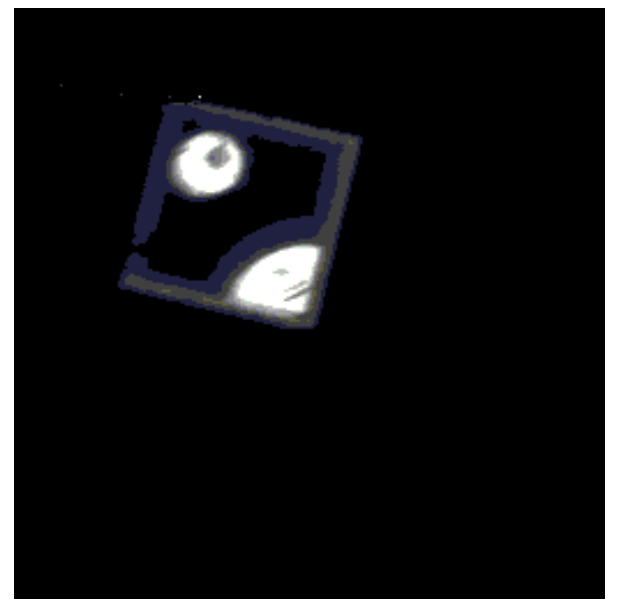

(b) 


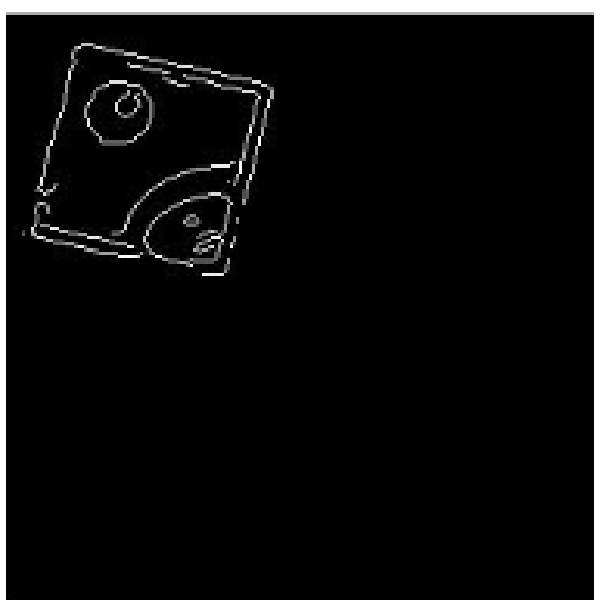

(c)

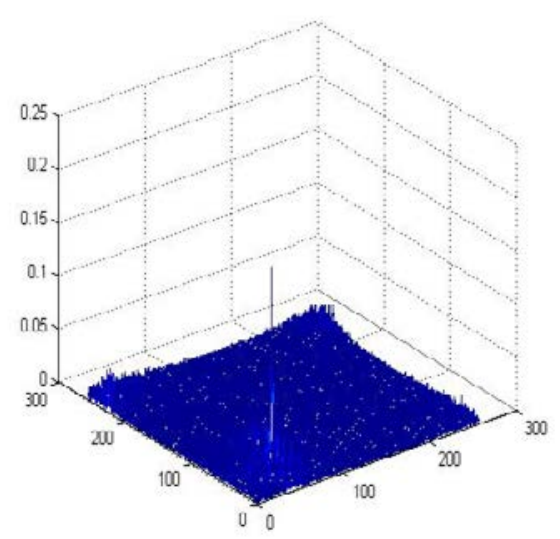

(e)

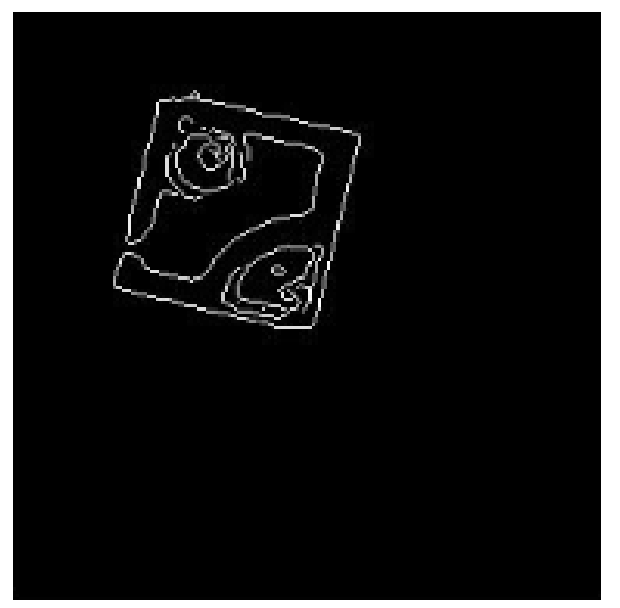

(d)

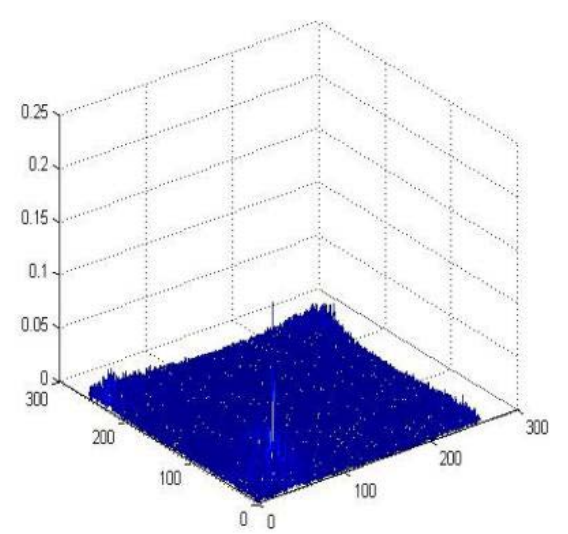

(f)

Fig.2. Result of edge phase correlation algorithm

In Fig.2, (a) and (b) are images to match, (c) and (d) are image derived from those images above using Canny edge detect. Image (e) is the result of phase correlation algorithm using all pixels in image (a) and (b). On the other hand, image (f) is the result of edge phase correlation algorithm using edge image in image (c) and (d). Fig.2 (g) is the time consumed in these two algorithms. As we can see, using edge phase correlation algorithm is using less time without changing the location result.

\section{Processing With Rotary}

But the above algorithm can handle image registration with pure translation, but for those situations that whose images contain translation and rotation, this algorithm is not applicable. For images with rotation and translation, there is relationship as following:

$$
\mathrm{R}(\mathrm{x}, \mathrm{y})=\mathrm{T}\left(\mathrm{x} \cos \theta_{0}+\mathrm{y} \sin \theta_{0}+\mathrm{x}_{0},-\mathrm{x} \sin \theta_{0}+\mathrm{y} \cos \theta_{0}+\mathrm{y}_{0}\right)
$$

After Fourier transform on both sides

$$
R_{f}(u, v)=e^{-j 2 \pi\left(u x_{0}+v y_{0}\right)} T_{f}\left(u \cos \theta_{0}+v \sin \theta_{0},-u \sin \theta_{0}+v \cos \theta_{0}\right)
$$

$\mathrm{T}_{\mathrm{f}}\left(\mathrm{u} \cos \theta_{0}+\mathrm{v} \sin \theta_{0},-\mathrm{u} \sin \theta_{0}+\mathrm{v} \cos \theta_{0}\right)$ has obvious meaning of the Fourier transform of template rotating $\theta_{0}$. Set a percentage here:

$$
\mathrm{G}(\mathrm{u}, \mathrm{v}, \theta)=\mathrm{R}_{\mathrm{f}}(\mathrm{u}, \mathrm{v}) / \mathrm{T}_{\mathrm{f}}(\mathrm{u} \cos \theta+\mathrm{v} \sin \theta,-\mathrm{u} \sin \theta+\mathrm{v} \cos \theta)
$$

Obviously if and only if $\theta=\theta_{0}, G(u, v, \theta)$ is an impulse function as formula, when the difference of $\theta$ and $\theta_{0}$, when the difference is increasing, the shape is less and less like impulse function. 


$$
G\left(u, v, \theta_{0}\right)=e^{-j 2 \pi\left(u x_{0}+v y_{0}\right)}
$$

\section{Curved Surface Fitting To Achieve Sub-Pixel Accuracy}

The essence of this method is to perform curved surface fitting with best matching point and its 8-neighborhood points, using value of correlation function matrix $\operatorname{CC}(\mathrm{x}, \mathrm{y})$ to make error between fitting surface and points mentioned above ${ }^{[7]}$. Then search fitting surface for the extreme value point, to obtain precise subpixel matching position.

The correlation function is expressed as quadric surface, the mathematical expression is:

$$
\mathrm{CC}(\mathrm{x}, \mathrm{y})=\mathrm{ax}^{2}+\mathrm{by}^{2}+\mathrm{cxy}+\mathrm{dx}+\mathrm{ey}+\mathrm{f}
$$

Set the peak point of $\mathrm{CC}(\mathrm{x}, \mathrm{y})$ as $\mathrm{CC}_{0}=\left(\mathrm{x}_{0}, \mathrm{y}_{0}\right)$, and take its surrounding points, respectively $\mathrm{CC}_{1}=\left(\mathrm{x}_{1}, \mathrm{y}_{1}\right), \mathrm{CC}_{2}=\left(\mathrm{x}_{2}, \mathrm{y}_{2}\right) \ldots . . \mathrm{CC}_{8}=\left(\mathrm{x}_{8}, \mathrm{y}_{8}\right)$, in neighborhood (including peak point itself) for fitting arithmetic. Process of quadratic curve fitting can be rewritten into the following linear equation in the form of matrix computation:

$$
\begin{aligned}
& A \xi=\eta \\
& \xi=\left(A^{T} A\right)^{-1} A^{T} \eta
\end{aligned}
$$

The meaning of the above matrix notations is followed:

$$
\begin{aligned}
A & =\left(\begin{array}{cccccc}
\mathrm{x}_{0}{ }^{2} & \mathrm{y}_{0}{ }^{2} & \mathrm{x}_{0} \mathrm{y}_{0} & \mathrm{x}_{0} & \mathrm{y}_{0} & 1 \\
\mathrm{x}_{1}{ }^{2} & \mathrm{y}_{1}{ }^{2} & \mathrm{x}_{1} \mathrm{y}_{1} & \mathrm{x}_{1} & \mathrm{y}_{1} & 1 \\
\cdots & \cdots & \cdots & \ldots & \ldots & \ldots \\
\mathrm{x}_{8}{ }^{2} & \mathrm{y}_{8}{ }^{2} & \mathrm{x}_{8} \mathrm{y}_{8} & \mathrm{x}_{8} & \mathrm{y}_{8} & 1
\end{array}\right) \\
\xi & =\left(\begin{array}{c}
\mathrm{a} \\
\mathrm{b} \\
\mathrm{c} \\
\mathrm{d} \\
\mathrm{e} \\
\mathrm{f}
\end{array}\right) \\
\eta & =\left(\begin{array}{c}
\mathrm{CC_{0 }} \\
\mathrm{CC}_{1} \\
\cdots \\
\mathrm{CC}_{8}
\end{array}\right)
\end{aligned}
$$

Notation $\xi$ can be obtained by solving formula (10). According to characteristic of quadratic surface, at the peak point surface derivative of each direction is zero, such as the formula (14). Therefore, solve the following equation formula (15) to get subpixel peak point.

$$
\begin{aligned}
& \left\{\begin{array}{l}
\frac{\partial z}{\partial x}=2 a x+c y+d \\
\frac{\partial z}{\partial y}=2 b y+c x+e
\end{array}\right. \\
& \left\{\begin{array}{l}
x=(2 d b-c e) /\left(c^{2}-4 a e\right) \\
y=(2 a e-d c) /\left(c^{2}-4 a e\right)
\end{array}\right.
\end{aligned}
$$




\section{IMPLEMENTATION}

Phase correlation algorithm is a kind of nonlinear, based on Fourier transform frequency domain correlation algorithm ${ }^{[6]}$. Since this method take power spectrum of image, so it can be robust of noise, illumination, but it is sensitive to image rotation. After summarizing the work of the predecessors, this paper proposes one kind of algorithm, by combining coarse positioning and precise positioning of two stages, to get the final match parameter. Coarse positioning and precise positioning will be discussed as followed in detail:

Coarse positioning: Perform optimized phase correlation algorithm to reference image and template image. After that, search for peaks of corresponding matric and map to the reference image, for purpose of obtaining the coarse positioning matching points, which mean coordinates of targets in reference image. Coarse matching work is done here, during which speed is priority, precision need not concern too much.

Precise matching phase: Select a point in a series of coarse matching points, cutting out an area of the neighborhood of the point which has the same size of template image (sometimes bigger), this area contains only one target, so as to simplify the matching problem, changing one-to-many matching problem into one-to-one. At this stage, the subpixel offsets and rotation angle are solved, both speed and accuracy should be concerned.

Algorithm mentioned above is designed as Fig.3 followed:

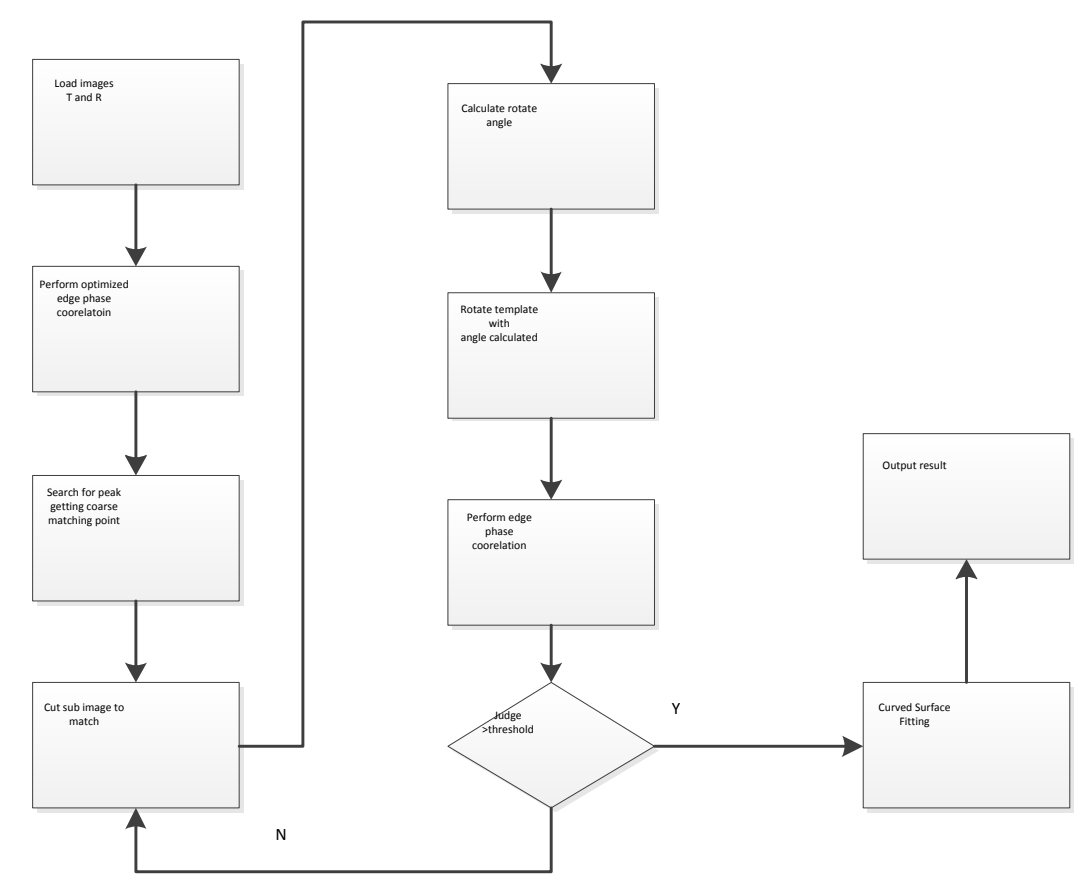

Fig.3. Outline of this algorithm

\section{Coarse Matching Process}

The algorithm is applied to recognize LED chip in automatic die bonder. From situation we encounter, some characteristic of location where LED chips lie can be observed. First of all, the angle between reference image and template image is usually less than 30 degree. Secondly, location of chips in reference image is usually uniformed. Thirdly, in images of LED, background and foreground can be easily separated.

Fig.4 (a) implies the reference image, (b) means the power spectrum of edge image to match, and one pulse can be seen in the matching position. But there are 4 targets here, that's the reason why the registration result is obviously wrong. It's because that when the reference image and the template image turned a relatively larger Angle, impulse function is not obvious. So tradition phase correlation algorithm need to be optimized to fit this situation.

Phase correlation function can be transformed like that: 


$$
\mathrm{CC}(\mathrm{u}, \mathrm{v})=\mathrm{R}_{\mathrm{f}}(\mathrm{u}, \mathrm{v}) * \mathrm{~T}_{\mathrm{f}}^{*}(\mathrm{u}, \mathrm{v})
$$

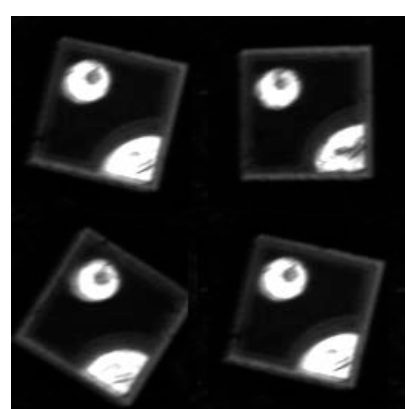

(a)

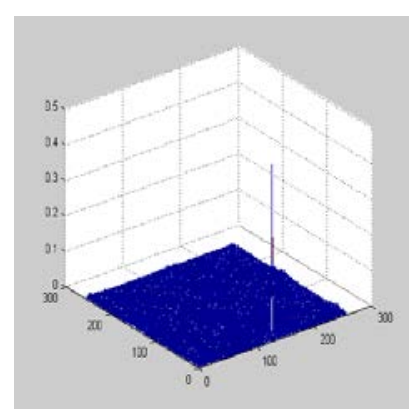

(b)

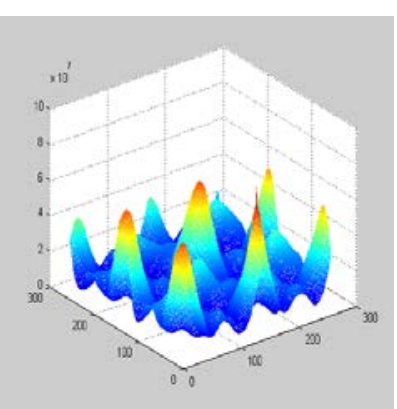

(c)

Fig.4. Result of optimized edge phase correlation algorithm

Fig.4 (c) is result with the formula (16), which improve feature of peak, with resistance of rotation in small range, meeting requirement of the expected. When angle difference between reference image and template is relatively small, peak corresponding is still sharp, but not so sharp as pulse function; when the difference gets larger, peak shape is still recognizable, which is not as shape as the one produce in above condition. In both condition mentioned above, location of target can be recognized, which means that formula (16) is an optimization for formula(3), not to mention significant reduction of the amount of time using in this algorithm.

In Fig.5 (b), red boxes are the position of sub image to be cut, according to location of peak position in figure 3(c). Location of the red boxes is basically right as seen, but still having a few pixels of error. It is because that phase correlation algorithm is sensitive to rotation, and presence of rotation will produce error. As the result that some parts of target are the outside of the box can be seen, we need to cut sub image a little larger than template, which is enough to include target. And then use these sub images to calculate rotate angle and subpixel coordinate. In Fig.4 (a), blue box is the actual place to cut off, as problem changes from a one-to-many matching into a one-to-one matching.

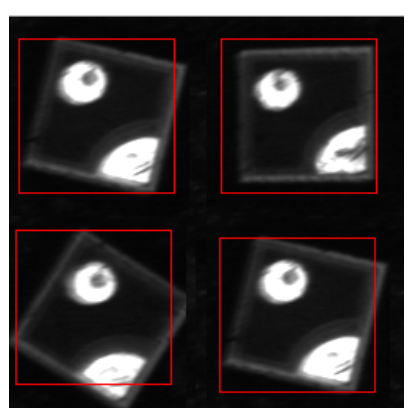

(a)

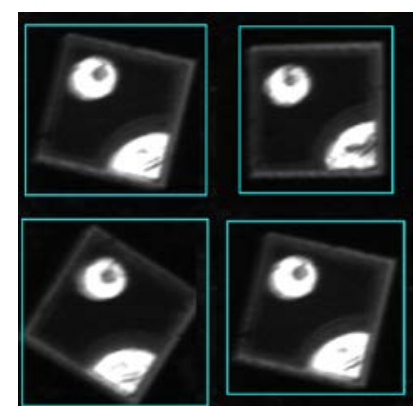

(b)

Fig.5. Sub image acquired coarse matching process

\section{Precise Matching Process}

After completion of the above steps, the coarse matching is completed. And one of sub image is chosen as a new reference image, for example, the target located in left top corner of the original reference image, as Fig.6 (a), is chosen to explain how the precise matching process works. Precise matching process between template image (Fig.6 (a)) and sub image(Fig.6 (b)) is demonstrated below.:

According to the formula (6), we know that the surface of $G(u, v, \theta)$ will change when $\theta$ varies. When $\theta$ gets more and more close to $\theta_{0}$, surface will become more and more like impulse function.
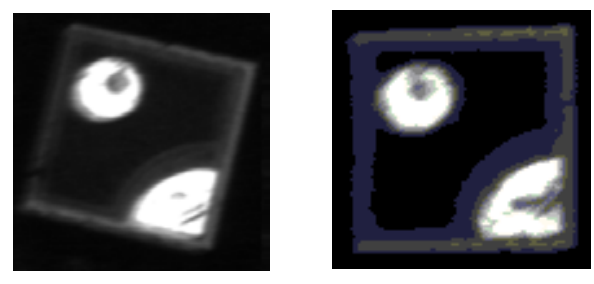
(a)

(b)

Fig.6. Images used in precise matching process

As the situation that angle difference is within small range is known, the template image rotates different angles respectively. With the change of the angle, different curved surface formed is obtained, as seen obviously in Fig.7. When $\theta=14^{\circ}$, power spectrum of both images is like a pulse function, reaching a highest extreme value, in accordance with the real value of angle difference $\theta=14^{\circ}$.

Higher angle precision can be done by rotate template with more angles. Here only for a reference image as an example, other sub image can also be located and recognized through the same steps.

Finally obtaining the matching of coordinates:

$$
\left(\mathrm{x}_{0}, \mathrm{y}_{0}\right)=\left(\Delta \mathrm{x}_{1}, \Delta \mathrm{y}_{1}\right)+\left(\Delta \mathrm{x}_{2}, \Delta \mathrm{y}_{2}\right)
$$

Displacement $\left(\Delta \mathrm{x}_{1}, \Delta \mathrm{y}_{1}\right)$ means the position where sub image located in reference image, and $\left(\Delta \mathrm{x}_{2}, \Delta \mathrm{y}_{2}\right)$ meaning the position where template image lies in sub image, which is the exact place where pulse belongs. To achieve the accuracy of subpixel level, neighborhood of the peak need to perform surface fitting and interpolation.

$\theta=14$

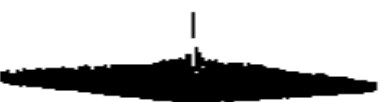

$\theta=13$

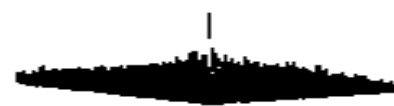

$\theta=12$

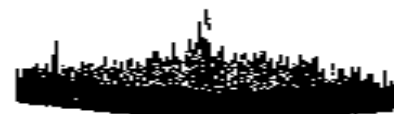

$\theta=11$

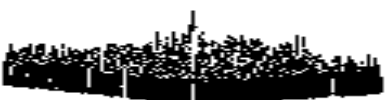

$\theta=15$

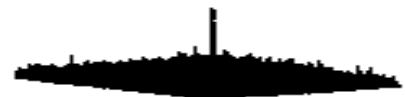

$\theta=16$

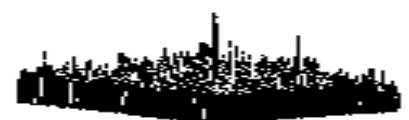

$\theta=17$

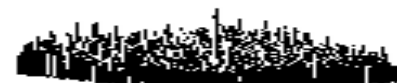

Fig.7. $G(u, v, \theta)$ changes with $\theta$ 


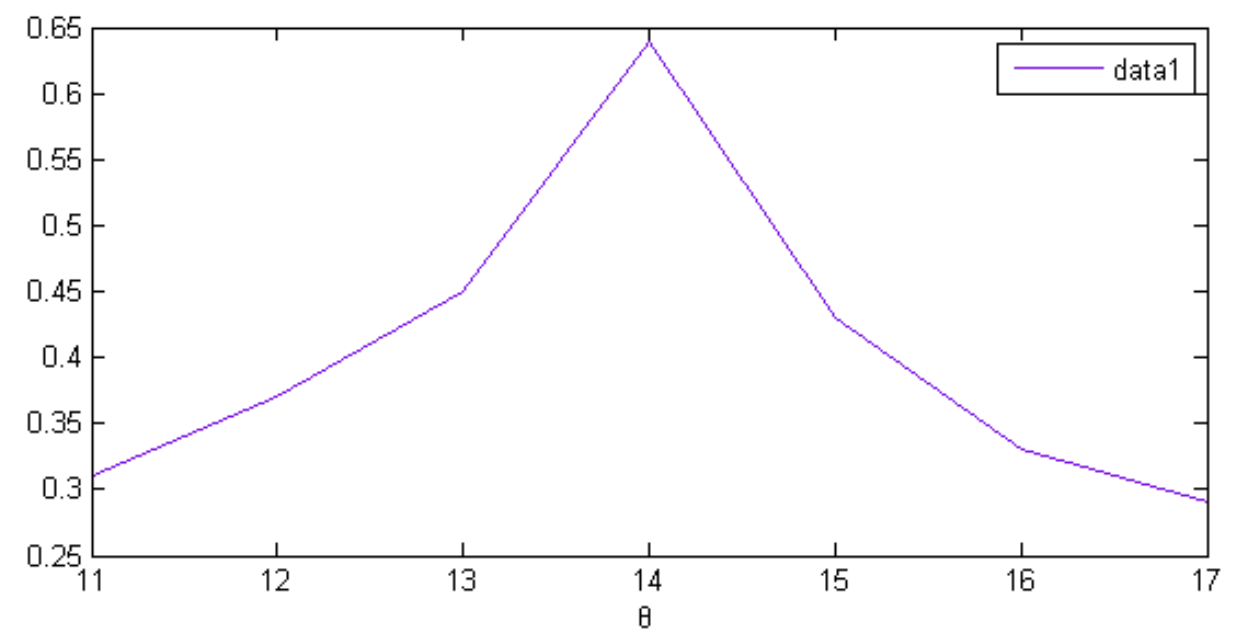

Fig.8. Peak of $G(u, v, \theta)$ change

In Fig.8, the angle difference between those images can be obtained. After rotating template with the angle $\theta=14^{\circ}$, and reusing edge phase correlation algorithm,peak point can be achieved at $(190,191)$, and perform curved surface fitting to its eight neighborhood, the following results are obtained.

\section{EXPERIMENTAL RESULTS}

The performance of this matching algorithm developed is tested on images in Fig.4 (a) and Fig.6 (b). Matches are considered valid only if the peak value of the IFFT is greater than 0.20. Theoretically, for exact matches this value should be equal to 1.0, however, the presence of dissimilar parts and difference of edge detect can reduce peak valve.

Match result is shown in table 1. Matching parameters are listed for the images mention above. Fig.9 shows the parameter calculated is very equal to actual result.

TABLE1.Matching result of translated and rotated image

\begin{tabular}{|l|l|l|l|l|}
\hline & 1 & 2 & 3 & 4 \\
\hline \multirow{3}{*}{ Theoretical result } & $(191.1,62.8)$ & $(190.4,191.2)$ & $(62.4,63.7)$ & $(61.7,195.3)$ \\
\cline { 2 - 5 } & $0 \circ$ & $14 \circ$ & $14 \circ$ & $34 \circ$ \\
\hline Calculated result & $(191.3,62.5)$ & $(190.3,190.8)$ & $(62.1,63.5)$ & $(61.7,195.8)$ \\
\cline { 2 - 6 } & $0 \circ$ & $14 \circ$ & $14 \circ$ & $34 \circ$ \\
\hline
\end{tabular}

The TABLE 1 shows that the result of this algorithm is satisfactory. Algorithm propose by this article is suitable for automatic die bonder.

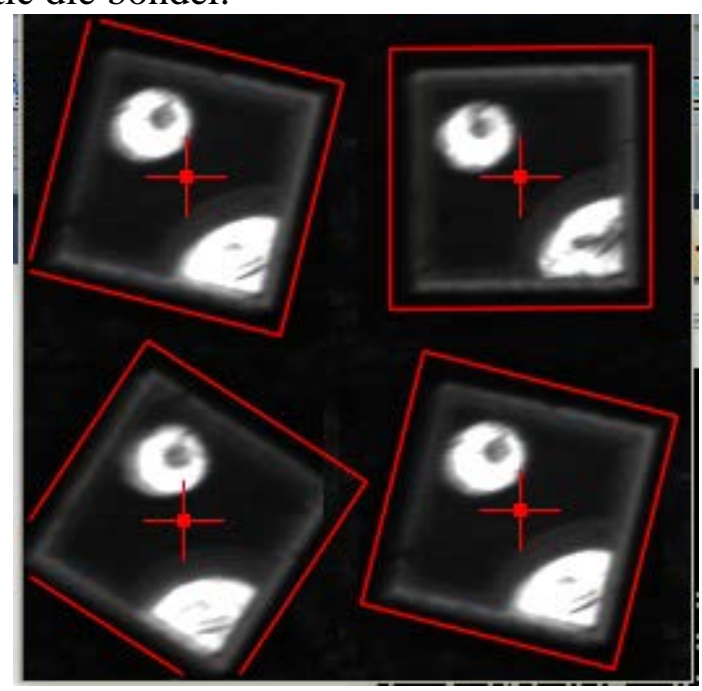

Fig.9. Location of registration target 


\section{References}

[1] E. D.Castro and C. Morandi. IEEE: TPAMI . Vol.9, No. 5(1987), pp. 700-703

[2] L.Lucchese and G.M.Cortelazzo. IEEE: Signal Processing. Vol.48, No. 6(2000), pp.1769-1786

[3] H.S.Stone and R.Wolpov. IEEE:TGRS. Vol.40, No3(2002), pp. 637-650

[4] Y.Keller, A.Averbuch and M.Israeli. IEEE: Image Processing. Vol.14, No. 1(2005), pp.12-22

[5] B.S.Reddy and B. N. Chatterji. IEEE:TIP. Vol.5, No.8 (1996) , pp. 1266-1271

[6] M.Morikawa, A.Katsumata, K.Kobayash. IEEE: Industrial Electronics.Vol.3(1999), pp.1208 $-1209$

[7] H.Xie, N.Hicks, G.R.Keller, H.Huang and V.Kernioviek. PERGAMON:Computers \& Geosciences. Vol.29(2003), pp.1045-1055

[8] P.Milanafr, Projection-based, frequency-domain estimation of superimposed translation motions. J.OPt.Soe.Am.Avol.13, No.11, pp. 2151-2162.1996.

[9] C. D. Kuglin and D. C. Hines,in: “The phase correlation image alignment method,” in Proc. IEEE 1975 Inf. Conf Cybernet. Society,New York ,NY, pp. 163-165. 